REVISTA PROYECCIONES $\mathrm{N}^{\circ} 10: 147-151$

Diciembre 1985 - ISSN 0716-0917

Jornada Matemáticas, Agosto 1985.

\title{
MEDIDAS FUZZY
}

HER IBERTO ROMAN*

ARTURO FLORES\%

\section{ABSTRACT.}

A partir de 1978 , con los trabajos de Zadeh, se empieza a desarro llar la llamada "Teoría Fuzzy".

En realidad, Zadeh crea una nueva concepción de la idea de "perte nencia a un conjunto", a partir de la cual se desarrolla toda una teoría de conjuntos $y$, por ende, toda una nueva matemática.

E1 camino recorrido desde entonces ha sido mucho y lo que nosotros deseamos en las próximas líneas es exponer en pequeño tópíco dentro de esta teoría y es lo referente a medidas e integrales Fuzzy.

Es en esta década en donde matemáticos tales como Sugeno (Japón) y y Ralescu (USA), aportan toda su creatividad para consolidar básicamente la teoría de integración Fuzzy.

* Departamento de Matemática, Facultad de Ciencias, Universidad de Tarapacá, Arica. 


\section{MEDIDAS FUZZY.}

Definición: Sea $\mathrm{X}$ un conjunto y $\mathcal{C}_{\text {una }} \sigma$ - álgebra de subconjuntos de $\mathrm{X}$. Diremos que la aplicación $\mu: \zeta \rightarrow[0, \infty]$ es una medida Fuzzy se verifica.

$\left.\mathrm{P}_{1}\right) \mu(\emptyset)=0$

$\left.\mathrm{P}_{2}\right) \quad \mathrm{A} C \mathrm{~B} \Rightarrow \mu(\mathrm{A}) \leqslant \mu(\mathrm{B})$

$\left.\mathrm{P}_{3}\right)$ Si $\mathrm{A}_{1} \mathrm{C} \mathrm{A}_{2} \mathrm{C} \ldots$ es una sucesión creciente en 4 , entonces $\mu\left(\sum_{n=1}^{\infty}\right.$ An $)=\lim _{n \rightarrow \infty} \mu($ An $)$

$\left.\mathrm{P}_{4}\right) \quad \mathrm{Si} \quad \mathrm{A}_{1} \quad \mathrm{~A}_{2} \quad \ldots$ es una sucesión decreciente en $\zeta \mathrm{y} \mu\left(\mathrm{A}_{1}\right)<\infty$, entonces $\mu(\underset{n=1}{\infty} A n)=\lim _{n \rightarrow \infty} \mu\left(A_{n}\right)$

Al triple $(X, \mathcal{F}, \mu)$ se le dice un Espacio de medida Fuzzy.

Algunos ejemplos:

1. En $\mathbb{R}$ la medida usual de Lebesgue es una medida Fuzzy.

2. Si $x=\{0,1,2, \ldots\}$ y $\Phi=P(x)$ entonces 1 a aplicación

$$
\mu(E)= \begin{cases}\sum_{i \in E} \frac{1}{2^{i+1}} & \text { Si } 0 \in E \\ l_{1} & \text { Si } 0 \in E \text { y } E-\{0\} \neq \emptyset \text { es una medida Fuzzy. } \\ \text { Si } E=\{0\} .\end{cases}
$$

Nota: Observemos que una medida fuzzy no necesita ser subaditiva ya que, en e1 ejemplo 2, vemos que:

Si $E_{1}=\{1,2, \ldots\}$ entonces $\mu\left(E_{1}\right)=\frac{1}{2}$

Si $E_{2}=\{0\}$ entonces $\mu\left(E_{2}\right)=1$ 


$$
\text { Ási } \mu\left(E_{1}\right)+\mu\left(E_{2}\right)=\frac{3}{2} \text { mientras que } \mu\left(E_{1} U E_{2}\right)=\infty \text {. }
$$

\section{LA INTEGRAL FUZZY.}

En lo que sigue f será una función medible no-negativa definida sobre.

Sugeno en su tesis doctoral (Tokyo) propone la siguiente defini ción para una integral Fuzzy.

Definición: Sea $A \in G$, entonces la integral Fuzzy de $f$ sobre A se define por:

$$
\begin{aligned}
& \int \mathrm{fdu}=\operatorname{Sup}_{\alpha \in[0, \infty]}[\operatorname{Inf}\{\alpha, \mu(\{\mathrm{A} \cap\{\dot{\mathrm{f}} \geq \alpha\}\} \\
& =\quad \mathrm{V}[\alpha \wedge \mu(\mathrm{A} \cap\{\mathrm{f} \geqslant \alpha\})] \\
& \\
& =\alpha \geqslant 0
\end{aligned}
$$

en donde $\{f \geqslant \alpha\}=\{x$ : $x: f(x) \geqslant \alpha\}$

Posteriormente Ralescu (1980) propone:

$$
\int f d u=V \quad f_{A} Q_{A}(s)
$$

En donde $S$ es una función simple menor o igual que $f$ y si $S=\sum a_{i} X_{A_{i}}$ entonces $Q_{A}(s)=\underset{1 \leqslant i \leqslant n}{\operatorname{Sup}}\left[\begin{array}{ll}a_{i} \wedge \mu(A & \left.\left.A_{i}\right)\right]\end{array}\right.$ 
Más aún, Ralescu y Adams (1981) proponen:

$$
\int_{A}^{f f d u}=A^{\prime} \in \operatorname{Sup}_{4}\left[\mu\left(A^{\prime}\right) \Lambda \operatorname{Inf}_{x \in A^{\prime}} f(x)\right]
$$

Lo sorprendente de todo esto es que:

Teorema: Las definiciones de Sugeno, Ralescu y Ralescu-A para la integral Fuzzy son equivalentes.

Definiciōn: (Función de rivel).

A la función $\mathrm{F}_{\mu}(\alpha)=\mu(\{f \geqslant \alpha\})$ le llamamos función de nivel corres pondiente a $\mathrm{f}$.

Es fácil ver que $F_{\mu}(\alpha)$ es monótona decreciente cualquiera sea $f$, $y$ que es valor de la integral Fuzzy $\int \mathrm{fdu}$ será justamente la abscisa del punto de intersección entre las gráficas de f y .

Ejemplo: $\underset{[0,1]}{\int x^{2} d u}=\frac{1}{2}$, en donde $\mu$ es la medida de Lebesgue.

OBS: Notemos que la integral Usual (en el sentido de Lebesgue) $\int \mathrm{x}^{2} \mathrm{du}=\frac{1}{3}$ 1o que significa una diferencia de $\frac{1}{6}$ de una con respecto a la otra. Este hecho Sugeno lo ataca a Fondo y el demuestra que esta diferencia, en valor absoluto, es a lo sumo $\frac{1}{4}$.

ALGUNAS PROPIEDADES DE LA INTEGRAL FUZZY.

F1) $\quad \int \mathrm{fdu}=\mathrm{f} \int_{\mathrm{A}} \mathrm{du}$

A

F2) Si a es constante, a $\in[0, \infty]$, entonces $\int a d u=a \wedge \mu(A)$. 
F3) i) $\quad f \leqslant g$ sobre $A \Rightarrow \int f d u \leqslant \int g d u$

A $\quad A$

ii) $A C B \Rightarrow \int f d u \leqslant \int f d u$

A $B$

F4) i) $\mu(A)=0 \Rightarrow \int f d u=0$

A

ii) $\int \mathrm{fdu}=0 \mathrm{~V}^{\prime} \mu(\{\mathrm{f}>0\} \cap \mathrm{A})=0$

A

F5) Si a constante no negativa

$$
\int(f+a) d u \leqslant f f d u+a \Lambda \mu(A)=\int f d u+\int a d u
$$

F6) $|f-g| \leqslant a \Rightarrow\left|\int f d u-\int g d u\right| \leqslant a$

Sobre A A A

Un estudio interesante es ahora obtener resultados análogos a los existentes en la teoría clásica de la medida, en lo que se refiere a la Convergencia de Integrales Fuzzy con respecto a la convergencia de sucesio nes de funciones, es decir, ¿Bajo qué condiciones $\int f_{n}$ du $\rightarrow \int f d u ~ s i f_{n} \rightarrow f$ sobre A?. 